\title{
An Ergodic Theorem for the Quantum Relative Entropy
}

\author{
Igor Bjelaković, Rainer Siegmund-Schultze* \\ Technische Universität Berlin \\ Fakultät II - Mathematik und Naturwissenschaften \\ Institut für Mathematik MA 7-2 \\ Straße des 17. Juni 13610623 Berlin, Germany
}

November 4, 2018

\begin{abstract}
We prove the ergodic version of the quantum Stein's lemma which was conjectured by Hiai and Petz. The result provides an operational and statistical interpretation of the quantum relative entropy as a statistical measure of distinguishability, and contains as a special case the quantum version of the Shannon-McMillan theorem for ergodic states. A version of the quantum relative Asymptotic Equipartition Property $(\mathrm{AEP})$ is given.
\end{abstract}

\section{Introduction}

This paper can be seen as an extension of the article [1] by Bjelaković, Krüger, Siegmund-Schultze and Szkoła, where instead of the von Neumann mean entropy of an ergodic quantum state $\psi$ on a quasilocal algebra $\mathcal{A}^{\infty}$, $\operatorname{dim} \mathcal{A}<\infty$, the mean relative entropy $s(\psi, \varphi)$ of $\psi$ with respect to some stationary product state $\varphi$ is the basic quantity. If we choose $\varphi$ to be the tracial state, the results here reduce to the quantum Shannon-McMillan theorem of 1. It turns out that the quantum mean relative entropy specifies the maximum exponential order, at which a typical subspace for the ergodic state $\psi$ becomes untypical for the product state $\varphi$. Typical subspaces which asymptotically attain this exponential order might be called maximally separating subspaces. In particular, it turns out that projectors onto maximally separating subspaces can be chosen in a way that an extended version of the quantum AEP is fulfilled: Each one-dimensional projector dominated by the maximally separating projector has an expected value with respect

\footnotetext{
*e-mail:\{igor, siegmund\}@math.tu-berlin.de
} 
to $\psi$ which is of an exponential order given by the von Neumann mean entropy of $\psi$ (in accordance with the quantum Shannon-McMillan theorem). The exponential order with respect to the reference state $\varphi$ is larger exactly by the relative entropy. This is a complete analogue to the classical situation, which is included in the result since $\mathcal{A}$ may be chosen abelian.

We point to the fact, that already in the classical situation the i.i.d. assumption concerning the reference state cannot be weakened very substantially, since there are examples with a reference process of very good mixing properties (B-process), but where a mean relative entropy does not even exist, see 8 .

The methods used to derive the results are mainly based on the techniques developed in the paper Hiai/Petz [3] and in [1. The Hiai/Petz paper considered the class of completely ergodic states $\psi$, and only an (exact) upper bound for the separation order was derived. Hiai and Petz were able to asymptotically reduce the problem to the abelian situation by constructing abelian sub-algebras with the remarkable property that they simultaneously allow a restriction of the two states $\psi, \varphi$ asymptotically without distortion of their mean entropies and mean relative entropy.

With some extension of the methods used already in [1] it was possible to drop the assumption of complete ergodicity as well as to show that the upper bound of the separation order is really a limit. This was conjectured already in Hiai/Petz 3 ] and proved for the case that both states are product (i.i.d.) states by Ogawa and Nagaoka in [4].

\section{Asymptotics of the Quantum Relative Entropy}

In this section we shall state our main result. We consider the lattice $\mathbb{Z}^{\nu}$. To each lattice site $\mathbf{x} \in \mathbb{Z}^{\nu}$ we assign a finite dimensional $C^{*}$-algebra $\mathcal{A}_{\mathbf{x}}$ being *-isomorphic to a fixed finite dimensional $C^{*}$-algebra $\mathcal{A}$. Recall that each finite dimensional $C^{*}$-algebra $\mathcal{A}$ can be thought of as $\bigoplus_{i=1}^{n} \mathcal{B}\left(\mathcal{H}_{i}\right)$ up to a *-isomorphism where the $\mathcal{H}_{i}$ are finite dimensional Hilbert spaces and $\mathcal{B}\left(\mathcal{H}_{i}\right)$ is the algebra of linear operators on $\mathcal{H}_{i}$. For a finite $\Lambda \subset \mathbb{Z}^{\nu}$ the algebra of local observables associated to $\Lambda$ is defined by

$$
\mathcal{A}_{\Lambda}:=\bigotimes_{\mathbf{x} \in \Lambda} \mathcal{A}_{\mathbf{x}}
$$

Clearly, this definition implies that for $\Lambda \subset \Lambda^{\prime}$ we have $\mathcal{A}_{\Lambda^{\prime}}=\mathcal{A}_{\Lambda} \otimes \mathcal{A}_{\Lambda^{\prime} \backslash \Lambda}$. Hence there is a canonical embedding of $\mathcal{A}_{\Lambda}$ into $\mathcal{A}_{\Lambda^{\prime}}$ given by $a \mapsto a \otimes \mathbf{1}_{\Lambda^{\prime} \backslash \Lambda}$ for $a \in \mathcal{A}_{\Lambda}$ where $\mathbf{1}_{\Lambda^{\prime} \backslash \Lambda}$ denotes the identity of $\mathcal{A}_{\Lambda^{\prime} \backslash \Lambda}$. The quasilocal $C^{*}$ algebra $\mathcal{A}^{\infty}$ is the norm completion of the normed algebra $\bigcup_{\Lambda \subset \mathbb{Z}^{\nu}} \mathcal{A}_{\Lambda}$ where the union is taken over all finite subsets $\Lambda$. For a precise definition of the quasilocal algebra we refer to [2] and [7]. The group $\mathbb{Z}^{\nu}$ acts in a natural way on the quasilocal algebra $\mathcal{A}^{\infty}$ as translations by $\mathbf{x} \in \mathbb{Z}^{\nu}$. A translation $T(\mathbf{x})$ 
by $\mathbf{x}$ associates to an $a \in \mathcal{A}_{\Lambda}$ the corresponding element $T(\mathbf{x}) a \in \mathcal{A}_{\Lambda+\mathbf{x}}$. This mapping $T(\mathbf{x})$ extends canonically to a $*$-automorphism on $\mathcal{A}^{\infty}$ (cf. [2], [7]).

A state $\psi$ on $\mathcal{A}^{\infty}$ is a linear, positive and unital mapping of $\mathcal{A}^{\infty}$ into $\mathbb{C}$. Each state $\psi$ on $\mathcal{A}^{\infty}$ corresponds to a compatible family $\left\{\psi^{(\Lambda)}\right\}_{\Lambda \subset \mathbb{Z}^{\nu}, \# \Lambda<\infty}$ where each $\psi^{(\Lambda)}$ denotes the restriction of $\psi$ to the local algebra $\mathcal{A}_{\Lambda}$. The compatibility means that $\psi^{\left(\Lambda^{\prime}\right)} \uparrow \mathcal{A}_{\Lambda}=\psi^{(\Lambda)}$ for $\Lambda \subset \Lambda^{\prime}$. A state $\psi$ is called stationary or translationally invariant if $\psi=\psi \circ T(\mathbf{x})$ for all $\mathbf{x} \in \mathbb{Z}^{\nu}$. The convex set of stationary states is denoted by $\mathcal{T}\left(\mathcal{A}^{\infty}\right)$ and is compact in the weak*-topology. The extremal points in $\mathcal{T}\left(\mathcal{A}^{\infty}\right)$ are the ergodic states. The basic theory of ergodic states is described in [2] and [7].

In this article the local sets $\Lambda$ will mainly be boxes in $\mathbb{Z}^{\nu}$ which are defined for $\mathbf{n}=\left(n_{1}, n_{2}, \ldots, n_{\nu}\right) \in \mathbb{N}^{\nu}$ by

$$
\Lambda(\mathbf{n}):=\left\{0, \ldots, n_{1}-1\right\} \times \ldots \times\left\{0, \ldots, n_{\nu}-1\right\},
$$

and the cubic boxes of edge length $n \in \mathbb{N}$ will be denoted by $\Lambda(n)$. The local algebras associated with $\Lambda(\mathbf{n})$ will be denoted by $\mathcal{A}^{(\mathbf{n})}$. In a similar fashion we will denote the restriction of a given state $\psi$ on $\mathcal{A}^{\infty}$ to the local algebra $\mathcal{A}^{(\mathbf{n})}$ by $\psi^{(\mathbf{n})}$. A state $\varphi$ is called a stationary product state if $\varphi^{(\mathbf{n})}=\left(\varphi^{(1)}\right)^{\otimes \Lambda(\mathbf{n})}$ holds for each $\mathbf{n} \in \mathbb{N}$.

The mean relative entropy of a stationary state $\psi$ with respect to the stationary product state $\varphi$ is defined by

$$
s(\psi, \varphi):=\lim _{\Lambda(\mathbf{n}) \nearrow \mathbb{N}^{\nu}} \frac{1}{\# \Lambda(\mathbf{n})} S\left(\psi^{(\mathbf{n})}, \varphi^{(\mathbf{n})}\right)=\sup _{\Lambda(\mathbf{n})} \frac{1}{\# \Lambda(\mathbf{n})} S\left(\psi^{(\mathbf{n})}, \varphi^{(\mathbf{n})}\right) .
$$

The last equation is an easy consequence of the superadditivity of the relative entropy of the state $\psi^{(\mathbf{n})}$ with respect to the product state $\varphi^{(\mathbf{n})}$. For the convenience of the reader we recall the definition of the relative entropy of the state $\sigma$ on a finite dimensional $C^{*}$-algebra $\mathcal{C}$ with respect to the state $\tau$ :

$$
S(\sigma, \tau):= \begin{cases}\operatorname{tr}\left(D_{\sigma}\left(\log D_{\sigma}-\log D_{\tau}\right)\right) & \text { if } \operatorname{supp}(\sigma) \leq \operatorname{supp}(\tau) \\ \infty & \text { otherwise }\end{cases}
$$

where $D_{\sigma}$ respectively $D_{\tau}$ denote the density operators of $\sigma$ respectively $\tau$ and $\operatorname{supp}(\sigma)$ is the support projector of the state $\sigma$. The important properties of the quantum relative entropy are summed up in the monograph [5] by Ohya and Petz. Later on we will need the notion of the mean relative entropy of the stationary state $\psi$ with respect to the stationary product state $\varphi$ on the lattice $G_{l}:=l \cdot \mathbb{Z}^{\nu}, l \geq 1$ an integer, given by

$$
s\left(\psi, \varphi, G_{l}\right):=\lim _{\Lambda(\mathbf{n}) \nearrow \mathbb{N}^{\nu}} \frac{1}{\# \Lambda(\mathbf{n})} S\left(\psi^{(l \mathbf{n})}, \varphi^{(l \mathbf{n})}\right)=l^{\nu} s(\psi, \varphi) .
$$

The basic quantity in this article is given by

$\beta_{\varepsilon, \mathbf{n}}(\psi, \varphi):=\min \left\{\log \varphi^{(\mathbf{n})}(q): q \in \mathcal{A}^{(\mathbf{n})}\right.$ projector and $\left.\psi^{(\mathbf{n})}(q) \geq 1-\varepsilon\right\}$, 
where $\varepsilon \in(0,1)$ and $\psi, \varphi \in \mathcal{T}\left(\mathcal{A}^{\infty}\right)$. The quantities appearing in (3) have a natural interpretation from the point of view of quantum statistical hypothesis testing. Assume that under the hypothesis $H_{1}$ the state of the system is given by $\psi^{(\mathbf{n})}$ and under the hypothesis $H_{2}$ the state is given by $\varphi^{(\mathbf{n})}$. A projector $q \in \mathcal{A}^{(\mathbf{n})}$ can be thought of as a decision rule: If a measurement of the projector $q$ on the system under consideration has outcome 1 , then the hypothesis $H_{1}$ is accepted and the measurement outcome 0 implies that $H_{2}$ is accepted. The quantities $\psi^{(\mathbf{n})}(1-q)$ and $\varphi^{(\mathbf{n})}(q)$ respectively describe the probabilities for the occurrence of the eigenvalue 0 respectively 1 if the hypothesis $H_{1}$ respectively $H_{2}$ was accepted, i.e. the error probabilities. The following theorem had been conjectured by Hiai and Petz in [3].

Theorem 2.1 Let $\psi$ be an ergodic state and let $\varphi$ be a stationary product state on $\mathcal{A}^{\infty}$ with mean relative entropy $s(\psi, \varphi)<\infty$. Then for all $\varepsilon \in(0,1)$ we have

$$
\lim _{\Lambda(\mathbf{n}) \nearrow \mathbb{N}^{\nu}} \frac{1}{\# \Lambda(\mathbf{n})} \beta_{\varepsilon, \mathbf{n}}(\psi, \varphi)=-s(\psi, \varphi) .
$$

The fact that in the case where both states are stationary product states the limit of $\frac{1}{\# \Lambda(\mathbf{n})} \beta_{\varepsilon, \mathbf{n}}(\psi, \varphi)$ exists and equals $-s(\psi, \varphi)$, had been shown by Ogawa and Nagaoka in 4. Their proof requires some deeper properties of so called quasi-entropies which are quantities defined analogously to the relative entropy where an arbitrary operator convex function is used in their definition instead of the function $-\log x$. We shall derive now the existence of the limit in the general case. As an instrument we will use some abelian approximation of all relevant quantities.

Projectors $q$ in (3) for which $\varphi^{(\mathbf{n})}(q)$ is of the order $e^{-\# \Lambda(\mathbf{n}) s(\psi, \varphi)}$ and for which $\psi(q) \geq 1-\varepsilon$ could be called maximally separating projectors and their range could be denoted as maximally separating subspace. The proof of this theorem reveals some additional information about maximally separating projectors, which is collected in the following

Theorem 2.2 (quantum relative AEP) Let $\psi$ be an ergodic state with mean entropy $s(\psi)$ and let $\varphi$ be a stationary product state on $\mathcal{A}^{\infty}$ with mean relative entropy $s(\psi, \varphi)<\infty$. Then for all $\varepsilon>0$ there is an $\mathbf{N}_{\varepsilon} \in \mathbb{N}^{\nu}$ such that for all $\mathbf{n} \in \mathbb{N}^{\nu}$ with $\Lambda(\mathbf{n}) \supseteq \Lambda\left(\mathbf{N}_{\varepsilon}\right)$ there exists an orthogonal projector $p_{\mathbf{n}}(\varepsilon) \in \mathcal{A}^{(\mathbf{n})}$ such that

1. $\psi^{(\mathbf{n})}\left(p_{\mathbf{n}}(\varepsilon)\right) \geq 1-\varepsilon$,

2. for all minimal projectors $p \in \mathcal{A}^{(\mathbf{n})}$ with $p \leq p_{\mathbf{n}}(\varepsilon)$ we have

$$
e^{-\#(\Lambda(\mathbf{n}))(s(\psi)+\varepsilon)}<\psi^{(\mathbf{n})}(p)<e^{-\#(\Lambda(\mathbf{n}))(s(\psi)-\varepsilon)},
$$

and consequently $e^{\#(\Lambda(\mathbf{n}))(s(\psi)-\varepsilon)}<\operatorname{tr}_{\mathbf{n}}\left(p_{\mathbf{n}}(\varepsilon)\right)<e^{\#(\Lambda(\mathbf{n}))(s(\psi)+\varepsilon)}$. 
3. for all minimal projectors $p \in \mathcal{A}^{(\mathbf{n})}$ with $p \leq p_{\mathbf{n}}(\varepsilon)$ we have

$$
e^{-\#(\Lambda(\mathbf{n}))(s(\psi)+s(\psi, \varphi)+\varepsilon)}<\varphi^{(\mathbf{n})}(p)<e^{-\#(\Lambda(\mathbf{n}))(s(\psi)+s(\psi, \varphi)-\varepsilon)},
$$

and consequently

$$
e^{-\#(\Lambda(\mathbf{n}))(s(\psi, \varphi)+\varepsilon)}<\varphi^{(\mathbf{n})}\left(p_{\mathbf{n}}(\varepsilon)\right)<e^{-\#(\Lambda(\mathbf{n}))(s(\psi, \varphi)-\varepsilon)},
$$

In the case that the state $\varphi$ is the tracial state on $\mathcal{A}^{\infty}$ Theorem 2.2 above is equivalent to the quantum version of the Shannon-McMillan theorem proved in [1] (cf. Theorem 2.1 in [1]).

The proof of Theorem 2.1 will make use of a classical law of large numbers for the (classical) mean relative entropy.

Theorem 2.3 Let $A$ be a finite set and $P$ respectively $Q$ be an ergodic respectively an i.i.d. probability measure on $\left[A^{\mathbb{Z}^{\nu}}, \mathfrak{A}^{\mathbb{Z}^{\nu}}\right]$, where $\mathfrak{A}^{\mathbb{Z}^{\nu}}$ is the $\sigma$ algebra generated by the cylinder sets. We have

$$
\lim _{\Lambda(\mathbf{n}) \nearrow \mathbb{N}^{\nu}} \frac{1}{\# \Lambda(\mathbf{n})} \log \frac{P^{(\mathbf{n})}\left(\omega_{\mathbf{n}}\right)}{Q^{(\mathbf{n})}\left(\omega_{\mathbf{n}}\right)}=D_{M}(P, Q) \quad P \text { - almost surely, }
$$

where $D_{M}(P, Q)$ denotes the mean relative entropy of $P$ with respect to $Q$ and $\omega_{\mathbf{n}} \in A^{\Lambda(\mathbf{n})}$ are the components of $\omega \in A^{\mathbb{Z}^{\nu}}$ corresponding to the box $\Lambda(\mathbf{n})$.

The proof of this classical assertion is an elementary application of the Shannon-McMillan-Breiman theorem and the individual ergodic theorem. The higher dimensional versions of these theorems needed in the present situation can be found in the article [6] by Ornstein and Weiss.

\section{Proof of the Main Theorems}

We start with the remark, that due to the assumption $s(\psi, \varphi)<\infty$ without any loss of generality we may assume the state $\varphi^{(1)}$ to be faithful. The following theorem and the Lemma 3.3 provide the tools to drop the condition of complete ergodicity that was originally used by Hiai and Petz to prove the assertion of Lemma 3.4. Then we make use of the Hiai/Petz approximation Theorem 3.2 to construct two stochastic processes which approximate the two states in the sense of entropy and relative entropy. The classical result Theorem 2.3 immediately yields a sequence of projectors which are separating with a rate given by the mean relative entropy. We still have to prove that this rate cannot be beaten. This is done in Lemma [3.6. The idea can be summarized as follows: Assume the existence of a better (higher rate) separating subspace. It is easy to see, that projecting this subspace into the constructed typical subspace would yield another separating subspace which would be 'better' than the constructed one. Now we make use of the classical relative AEP valid for the constructed subspace to exclude this possibility. 
Theorem 3.1 Let $\psi$ be an ergodic state on $\mathcal{A}^{\infty}$. Then for every subgroup $G_{l}:=l \cdot \mathbb{Z}^{\nu}$, with $l>1$ an integer, there exists a $\mathbf{k}(l) \in \mathbb{N}^{\nu}$ and a unique convex decomposition of $\psi$ into $G_{l}$-ergodic states $\psi_{\mathbf{x}}$ :

$$
\psi=\frac{1}{\# \Lambda(\mathbf{k}(l))} \sum_{\mathbf{x} \in \Lambda(\mathbf{k}(l))} \psi_{\mathbf{x}} .
$$

The $G_{l}$-ergodic decomposition (5) has the following properties:

1. $k_{j}(l) \leq l$ and $k_{j}(l) \mid l$ for all $j \in\{1, \ldots, \nu\}$

2. $\left\{\psi_{\mathbf{x}}\right\}_{\mathbf{x} \in \Lambda(\mathbf{k}(l))}=\left\{\psi_{0} \circ T(-\mathbf{x})\right\}_{\mathbf{x} \in \Lambda(\mathbf{k}(l))}$

3. For every $G_{l}$-ergodic state $\psi_{\mathbf{x}}$ in the convex decomposition (5) of $\psi$ the mean entropy with respect to $G_{l}, s\left(\psi_{\mathbf{x}}, G_{l}\right)$, is equal to the mean entropy $s\left(\psi, G_{l}\right)$, i.e.

$$
s\left(\psi_{\mathbf{x}}, G_{l}\right)=s\left(\psi, G_{l}\right)
$$

for all $\mathbf{x} \in \Lambda(\mathbf{k}(l))$.

4. For each $G_{l}$-ergodic state $\psi_{\mathbf{x}}$ in the convex decomposition (5) of $\psi$ and for every stationary product state $\varphi$ the mean relative entropy with respect to $G_{l}, s\left(\psi_{\mathbf{x}}, \varphi, G_{l}\right)$, fulfills

$$
s\left(\psi_{\mathbf{x}}, \varphi, G_{l}\right)=s\left(\psi, \varphi, G_{l}\right)
$$

for all $\mathbf{x} \in \Lambda(\mathbf{k}(l))$.

Proof of Theorem 3.1; The first three items have been established in 1], Theorem 3.1.

The proof of the last item is based on the monotonicity of the relative entropy and the usage of item 2. of the theorem. For each $\mathbf{n} \in \mathbb{N}^{\nu}$ and $\mathbf{x} \in \Lambda(\mathbf{k}(l))$ we have $\psi_{\mathbf{x}}^{(\Lambda(l \mathbf{n}))}=\psi_{0}^{(\Lambda(l \mathbf{n})-\mathbf{x})}$ by the second item. We consider the box $\tilde{\Lambda}(\mathbf{n})$ containing $\Lambda(l \mathbf{n})$ and each $\Lambda(l \mathbf{n})-\mathbf{x}$ defined by

$$
\tilde{\Lambda}(\mathbf{n}):=\left\{-l, \ldots, \ln _{1}-1\right\} \times \ldots \times\left\{-l, \ldots, l n_{\nu}-1\right\},
$$

and the box $\hat{\Lambda}(\mathbf{n})$ contained in $\Lambda(l \mathbf{n})$ and $\Lambda(l \mathbf{n})-\mathbf{x}$ given by

$$
\hat{\Lambda}(\mathbf{n}):=\left\{0, \ldots, l\left(n_{1}-1\right)-1\right\} \times \ldots \times\left\{0, \ldots, l\left(n_{\nu}-1\right)-1\right\} .
$$

The volumes of these boxes are asymptotically equivalent in the sense that the quotient tends to one. Hence using the observation above and twice the monotonicity of the relative entropy we obtain

$$
\begin{aligned}
S\left(\psi_{0}^{(\tilde{\Lambda}(\mathbf{n}))}, \varphi^{(\tilde{\Lambda}(\mathbf{n}))}\right) & \geq S\left(\psi_{0}^{(\Lambda(l \mathbf{n})-\mathbf{x})}, \varphi^{(\Lambda(l \mathbf{n})-\mathbf{x})}\right) \\
& =S\left(\psi_{\mathbf{x}}^{(\Lambda(l \mathbf{n}))}, \varphi^{(\Lambda(l \mathbf{n})-\mathbf{x})}\right) \\
& \geq S\left(\psi_{\mathbf{x}}^{(\hat{\Lambda}(\mathbf{n}))}, \varphi^{(\hat{\Lambda}(\mathbf{n}))}\right) .
\end{aligned}
$$


After dividing by $\# \Lambda(\mathbf{n})$ and taking the limit $\Lambda(\mathbf{n}) \nearrow \mathbb{N}^{\nu}$ this inequality chain shows that

$$
s\left(\psi_{0}, \varphi, G_{l}\right) \geq s\left(\psi_{\mathbf{x}}, \varphi, G_{l}\right)
$$

holds. A similar argument using $\psi_{\mathbf{x}}^{(\Lambda(l \mathbf{n})+\mathbf{x})}=\psi_{0}^{(\Lambda(l \mathbf{n}))}$ shows that the reverse inequality is also valid. Hence we established

$$
s\left(\psi_{0}, \varphi, G_{l}\right)=s\left(\psi_{\mathbf{x}}, \varphi, G_{l}\right) .
$$

Since the mean relative entropy is affine in its first argument on the set of $G_{l}$-invariant states this implies

$$
s\left(\psi_{\mathbf{x}}, \varphi, G_{l}\right)=s\left(\psi, \varphi, G_{l}\right)=l^{\nu} s(\psi, \varphi) .
$$

An important ingredient in our proof of the Theorem 2.1 is a result proved by Hiai and Petz in [3]. The starting point is the spectral decomposition of the density operator $D_{\varphi^{(1)}}$ corresponding to the state $\varphi^{(1)}$ on $\mathcal{A}$ :

$$
D_{\varphi(1)}=\sum_{i=1}^{d} \lambda_{i} e_{i}
$$

where $e_{i}$ are one-dimensional projectors. Clearly, the spectral representation of the tensor product of $D_{\varphi^{(1)}}$ over a box $\Lambda(\mathbf{y})$ can be written as

$$
D_{\varphi(\mathbf{y})}=\sum_{i_{1}, \ldots, i_{N(\mathbf{y})}=1}^{d} \lambda_{i_{1}} \cdots \lambda_{i_{N(\mathbf{y})}} e_{i_{1}} \otimes \ldots \otimes e_{i_{N(\mathbf{y})}},
$$

where we have chosen some enumeration $\{1, \ldots, N(\mathbf{y})\}$ of the points belonging to the box $\Lambda(\mathbf{y})$. We have $N(\mathbf{y})=y_{1} \cdot \ldots \cdot y_{\nu}$. Collecting all one dimensional projectors $e_{i_{1}} \otimes \ldots \otimes e_{i_{N(\mathbf{y})}}$ which correspond to the same eigenvalue of $D_{\varphi(\mathrm{y})}$ we can rewrite the last expression as

$$
D_{\varphi(\mathbf{y})}=\sum_{n_{1}, \ldots n_{d}: n_{1}+\ldots+n_{d}=N(\mathbf{y})}\left(\prod_{k=1}^{d} \lambda_{k}^{n_{k}}\right) p_{n_{1}, \ldots n_{d}},
$$

with

$$
p_{n_{1}, \ldots n_{d}}:=\sum_{\left(i_{1}, \ldots, i_{N(\mathbf{y})}\right) \in I_{n_{1}, \ldots n_{d}}} e_{i_{1}} \otimes \ldots \otimes e_{i_{N(\mathbf{y})}},
$$

where

$$
I_{n_{1}, \ldots n_{d}}:=\left\{\left(i_{1}, \ldots, i_{N(\mathbf{y})}\right): \#\left\{j: i_{j}=k\right\}=n_{k} \text { for } 1 \leq k \leq d\right\} .
$$

We define the conditional expectation with respect to the trace by

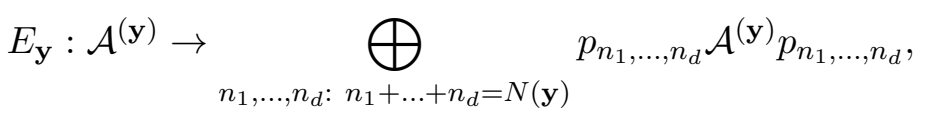




$$
E_{\mathbf{y}}(a):=\sum_{n_{1}, \ldots, n_{d}: n_{1}+\ldots+n_{d}=N(\mathbf{y})} p_{n_{1}, \ldots, n_{d}} a p_{n_{1}, \ldots, n_{d}} .
$$

We are prepared to state the announced important result of Hiai and Petz, Lemma 3.1 and Lemma 3.2 in [3].

Theorem 3.2 If $\psi$ is a stationary state on $\mathcal{A}^{\infty}$ and $\mathcal{D}_{\mathbf{y}}$ is the abelian subalgebra of $\mathcal{A}^{(\mathbf{y})}$ generated by $\left\{p_{n_{1}, \ldots n_{d}} D_{\psi(\mathbf{y})} p_{n_{1}, \ldots n_{d}}\right\}_{n_{1}, \ldots, n_{d}} \cup\left\{p_{n_{1}, \ldots n_{d}}\right\}_{n_{1}, \ldots, n_{d}}$ then

$$
S\left(\psi^{(\mathbf{y})}, \varphi^{(\mathbf{y})}\right)=S\left(\psi^{(\mathbf{y})} \uparrow \mathcal{D}_{\mathbf{y}}, \varphi^{(\mathbf{y})} \uparrow \mathcal{D}_{\mathbf{y}}\right)+S\left(\psi^{(\mathbf{y})} \circ E_{\mathbf{y}}\right)-S\left(\psi^{(\mathbf{y})}\right),
$$

and

$$
S\left(\psi^{(\mathbf{y})} \circ E_{\mathbf{y}}\right)-S\left(\psi^{(\mathbf{y})}\right) \leq d \log (\# \Lambda(\mathbf{y})+1) .
$$

Consequently we have

$$
\lim _{\Lambda(\mathbf{y}) \nearrow \mathbb{N}^{\nu}} \frac{1}{\# \Lambda(\mathbf{y})} S\left(\psi^{(\mathbf{y})} \uparrow \mathcal{D}_{\mathbf{y}}, \varphi^{(\mathbf{y})} \uparrow \mathcal{D}_{\mathbf{y}}\right)=s(\psi, \varphi) .
$$

Remark: This theorem had been proved by Hiai and Petz in [3] for the onedimensional lattice. However, their proof extends canonically to the present situation.

Any abelian algebra $\mathcal{D}_{\mathbf{y}}$ in Theorem 3.2 can be represented as

$$
\mathcal{D}_{\mathbf{y}}=\bigoplus_{i=1}^{a_{\mathbf{y}}} \mathbb{C} \cdot f_{\mathbf{y}, i}
$$

where $\left\{f_{\mathbf{y}, i}\right\}_{i=1}^{a_{\mathbf{y}}}$ is the set of orthogonal minimal projectors in $\mathcal{D}_{\mathbf{y}}$. For any $\mathbf{y}$ we introduce a maximally abelian refinement $\mathcal{B}_{\mathbf{y}}$ of $\mathcal{D}_{\mathbf{y}}$ by splitting each $f_{\mathbf{y}, i}$ into a sum of orthogonal and minimal (in the sense of the algebra $\mathcal{A}^{(\mathbf{y})}$ ) projectors $g_{\mathbf{y}, i, j}$ which leads to the representation

$$
\mathcal{B}_{\mathbf{y}}=\bigoplus_{i=1}^{a_{\mathbf{y}}} \bigoplus_{j=1}^{b_{\mathbf{y}, i}} \mathbb{C} \cdot g_{\mathbf{y}, i, j}
$$

By the monotonicity of the relative entropy we get

$$
S\left(\psi^{(\mathbf{y})} \uparrow \mathcal{D}_{\mathbf{y}}, \varphi^{(\mathbf{y})} \uparrow \mathcal{D}_{\mathbf{y}}\right) \leq S\left(\psi^{(\mathbf{y})} \uparrow \mathcal{B}_{\mathbf{y}}, \varphi^{(\mathbf{y})} \uparrow \mathcal{B}_{\mathbf{y}}\right) \leq S\left(\psi^{(\mathbf{y})}, \varphi^{(\mathbf{y})}\right),
$$

from which we deduce

$$
\lim _{\Lambda(\mathbf{y}) \nearrow_{\mathbb{N}^{\nu}}} \frac{1}{\# \Lambda(\mathbf{y})} S\left(\psi ^ { ( \mathbf { y } ) } \left\lceil\mathcal{B}_{\mathbf{y}}, \varphi^{(\mathbf{y})}\left\lceil\mathcal{B}_{\mathbf{y}}\right)=s(\psi, \varphi)\right.\right.
$$

by (11).

We choose a positive integer $l$ and consider the decomposition of $\psi \in$ 
$\partial_{\text {ex }} \mathcal{T}\left(\mathcal{A}^{\infty}\right)$ into states $\psi_{\mathbf{x}}$ being ergodic with respect to the action of $G_{l}$, i.e. $\psi=\frac{1}{\# \Lambda(\mathbf{k}(l))} \sum_{\mathbf{x} \in \Lambda(\mathbf{k}(l))} \psi_{\mathbf{x}}$ in accordance with Theorem 3.1 Moreover, we consider a stationary product state $\varphi$. Note that this state is $G_{l}$-ergodic for each $l \in \mathbb{Z}$. In order to keep our notation transparent we agree on following abbreviations:

$$
s:=s\left(\psi, \varphi, \mathbb{Z}^{\nu}\right)=s(\psi, \varphi)
$$

i.e. the mean relative entropy of the state $\psi$ computed with respect to $\mathbb{Z}^{\nu}$. We write $\mathcal{B}_{l}$ for $\mathcal{B}_{(l, l, \ldots, l)}$ and set

$$
s_{\mathbf{x}}^{(l)}:=\frac{1}{\# \Lambda(l)} S\left(\psi_{\mathbf{x}}^{(\Lambda(l))} \uparrow \mathcal{B}_{l}, \varphi^{(\Lambda(l))} \uparrow \mathcal{B}_{l}\right)
$$

and

$$
s^{(l)}:=\frac{1}{\# \Lambda(l)} S\left(\psi^{(\Lambda(l))} \uparrow \mathcal{B}_{l}, \varphi^{(\Lambda(l))} \uparrow \mathcal{B}_{l}\right) .
$$

From the Theorem 3.1 above we know that

$$
s\left(\psi_{\mathbf{x}}, \varphi, G_{l}\right)=s\left(\psi, \varphi, G_{l}\right)=l^{\nu} \cdot s(\psi, \varphi), \quad \forall \mathbf{x} \in \Lambda(\mathbf{k}(l)) .
$$

For an arbitrarily chosen $\eta>0$ let us define the set

$$
A_{l, \eta}:=\left\{\mathbf{x} \in \Lambda(\mathbf{k}(l)): s_{\mathbf{x}}^{(l)}<s-\eta\right\} .
$$

By $A_{l, \eta}^{c}$ we denote its complement. In the next lemma we shall show that the essential part of $G_{l}$-ergodic components of $\psi$ have the entropy per site close to $s$ as $l$ becomes large, even if we restrict them to the abelian algebras $\mathcal{B}_{l}$.

Lemma 3.3 If $\psi$ is a $\mathbb{Z}^{\nu}$-ergodic state and $\varphi$ a stationary product state on $\mathcal{A}^{\infty}$ and if $s(\psi, \varphi)<\infty$, then

$$
\lim _{l \rightarrow \infty} \frac{\# A_{l, \eta}}{\# \Lambda(\mathbf{k}(l))}=0
$$

holds for every $\eta>0$.

Proof of Lemma 3.3: We suppose, on the contrary, that there exists some $\eta_{0}>0$ such that $\lim \sup _{l} \frac{\# A_{l, \eta_{0}}}{\# \Lambda(\mathbf{k}(l))}=a>0$. Then we can select a subsequence, which we denote again by $(l)$ for simplicity, with the property

$$
\lim _{l \rightarrow \infty} \frac{\# A_{l, \eta_{0}}}{\# \Lambda(\mathbf{k}(l))}=a .
$$


Using joint convexity of the relative entropy we have the following estimate:

$$
\begin{aligned}
\# \Lambda(\mathbf{k}(l)) \cdot s^{(l)} & \leq \sum_{\mathbf{x} \in \Lambda(\mathbf{k}(l))} s_{\mathbf{x}}^{(l)} \\
& =\sum_{\mathbf{x} \in A_{l, \eta_{0}}} s_{\mathbf{x}}^{(l)}+\sum_{\mathbf{x} \in A_{l, \eta_{0}}^{c}} s_{\mathbf{x}}^{(l)} \\
& \stackrel{17}{<} \# A_{l, \eta_{0}} \cdot\left(s-\eta_{0}\right)+\# A_{l, \eta_{0}}^{c} \cdot \max _{\mathbf{x} \in A_{l, \eta_{0}}^{c}} s_{\mathbf{x}}^{(l)} .
\end{aligned}
$$

Employing that for the mean entropy

$$
s_{\mathbf{x}}^{(l)} \leq \frac{1}{\# \Lambda(l)} S\left(\psi_{\mathbf{x}}^{(\Lambda(l))}, \varphi^{(\Lambda(l))}\right) \quad \text { (by the monotonicity) }
$$

and

$$
\begin{aligned}
s\left(\psi_{\mathbf{x}}, \varphi, G_{l}\right) & =\lim _{\Lambda(\mathbf{m}) \nearrow \mathbb{N}^{\nu}} \frac{1}{\# \Lambda(\mathbf{m})} S\left(\psi_{\mathbf{x}}^{(l \mathbf{m})}, \varphi^{(l \mathbf{m})}\right) \\
& =\sup _{\Lambda(\mathbf{m})} \frac{1}{\# \Lambda(\mathbf{m})} S\left(\psi_{\mathbf{x}}^{(l \mathbf{m})}, \varphi^{(l \mathbf{m})}\right)
\end{aligned}
$$

are fulfilled, we obtain an upper bound for the last term in (18):

$$
\begin{aligned}
\# A_{l, \eta_{0}}^{c} \cdot \max _{\mathbf{x} \in A_{l, \eta_{0}}^{c}} s_{\mathbf{x}}^{(l)} & \leq \# A_{l, \eta_{0}}^{c} \cdot \max _{\mathbf{x} \in A_{l, \eta_{0}}^{c}} \frac{1}{l^{\nu}} s\left(\psi_{\mathbf{x}}, \varphi, G_{l}\right) \\
& =\# A_{l, \eta_{0}}^{c} \cdot s(\psi, \varphi) \quad(\operatorname{by}(\underline{16}) .
\end{aligned}
$$

Inserting this in (18) and dividing by $\# \Lambda(\mathbf{k}(l))$ we obtain

$$
s^{(l)}<\frac{\# A_{l, \eta_{0}}}{\# \Lambda(\mathbf{k}(l))}\left(s-\eta_{0}\right)+\frac{\# A_{l, \eta_{0}}^{c}}{\# \Lambda(\mathbf{k}(l))} s .
$$

And after taking limits we arrive at the following contradictory inequality:

$$
s \leq a\left(s-\eta_{0}\right)+(1-a) s=s-a \eta_{0}<s,
$$

since $\lim _{l \rightarrow \infty} s^{(l)}=s$ by Theorem 3.2 and $s<\infty$. Hence $a=0$.

Lemma 3.4 Let $\psi$ be an ergodic state on $\mathcal{A}^{\infty}$ and let $\varphi$ be a stationary product state on $\mathcal{A}^{\infty}$ fulfilling $s(\psi, \varphi)<\infty$. Then for every $\varepsilon \in(0,1)$

$$
\limsup _{\Lambda(\mathbf{n}) \nearrow \mathbb{N}^{\nu}} \frac{1}{\# \Lambda(\mathbf{n})} \beta_{\varepsilon, \mathbf{n}}(\psi, \varphi) \leq-s(\psi, \varphi) .
$$


Proof of Lemma 3.4: We fix $\varepsilon>0$ and choose arbitrary $\eta, \delta>0$. Consider the $G_{l}$-ergodic decomposition

$$
\psi=\frac{1}{\# \Lambda(\mathbf{k}(l))} \sum_{\mathbf{x} \in \Lambda(\mathbf{k}(l))} \psi_{\mathbf{x}}
$$

of $\psi$ for integers $l \geq 1$. By Lemma 3.3 there is an integer $L \geq 1$ such that for any $l \geq L$

$$
\frac{\varepsilon}{2} \geq \frac{1}{\# \Lambda(\mathbf{k}(l))} \# A_{l, \eta} \geq 0
$$

holds, where $A_{l, \eta}$ is defined by (17). This inequality implies

$$
\frac{1}{\# \Lambda(\mathbf{k}(l))} \# A_{l, \eta}^{c} \cdot\left(1-\frac{\varepsilon}{2}\right) \geq 1-\varepsilon .
$$

Recall that by definition of $A_{l, \eta}$ we have

$$
\frac{1}{\# \Lambda(l)} S\left(\psi_{\mathbf{x}}^{(\Lambda(l))} \uparrow \mathcal{B}_{l}, \varphi^{(\Lambda(l))} \uparrow \mathcal{B}_{l}\right) \geq s(\psi, \varphi)-\eta \quad \text { for all } \mathbf{x} \in A_{l, \eta}^{c} .
$$

We fix an $l \geq L$ and consider the abelian quasi-local $C^{*}$-algebra $\mathcal{B}_{l}^{\infty}$ built up from $\mathcal{B}_{l} . \mathcal{B}_{l}^{\infty}$ is clearly a $C^{*}$-subalgebra of $\mathcal{A}^{\infty}$. We set

$$
m_{\mathbf{x}}:=\psi_{\mathbf{x}}\left\lceil\mathcal{B}_{l}^{\infty} \text { and } m_{\mathbf{x}}^{(\mathbf{n})}:=\psi_{\mathbf{x}}\left\lceil\mathcal{B}_{l}^{(\mathbf{n})}\right. \text {. }\right.
$$

Moreover we define

$$
p:=\varphi \uparrow \mathcal{B}_{l}^{\infty} \text { and } p^{(\mathbf{n})}:=\varphi \uparrow \mathcal{B}_{l}^{(\mathbf{n})} .
$$

The state $p$ is a $G_{l}$-stationary product state. On the other hand, Theorem 4.3.17. in 2] shows that the states $m_{\mathbf{x}}$ are $G_{l}$-ergodic. Due to the Gelfand isomorphism and the Riesz representation theorem we can (and shall) identify all the states above with the probability measures on the corresponding maximal ideal space of $\mathcal{B}_{l}^{\infty}$. Since the algebra $\mathcal{B}_{l}$ is abelian and finite dimensional this compact maximal ideal space can be thought of as $B_{l}^{\mathbb{Z}^{\nu}}$ for an appropriately chosen finite set $B_{l}$. This is essentially the well-known Kolmogorov representation of a classical dynamical system.

By the definition of the measures $m_{\mathbf{x}}$ and $p$, monotonicity of the relative entropy and the fourth item in Theorem 3.1 we have

$$
D_{M}\left(m_{\mathbf{x}}, p\right) \leq s\left(\psi_{\mathbf{x}}, \varphi, G_{l}\right)=l^{\nu} s(\psi, \varphi)<\infty,
$$

where $D_{M}\left(m_{\mathbf{x}}, p\right)$ denotes the mean relative entropy of $m_{\mathbf{x}}$ with respect to $p$. Using the Theorem 2.3 we see that

$$
\lim _{\Lambda(\mathbf{n}) \nearrow_{\mathbb{N}^{\nu}}} \frac{1}{\Lambda(\mathbf{n})} \log \frac{m_{\mathbf{x}}^{(\mathbf{n})}\left(\omega_{\mathbf{n}}\right)}{p^{(\mathbf{n})}\left(\omega_{\mathbf{n}}\right)}=D_{M}\left(m_{\mathbf{x}}, p\right)=: D_{M, \mathbf{x}}
$$


$m_{\mathbf{x}}$-almost surely for all $\mathbf{x} \in \Lambda(\mathbf{k}(l))$, where $\omega_{\mathbf{n}} \in B_{l}^{\Lambda(\mathbf{n})}$ are the components of $\omega \in B_{l}^{\mathbb{Z}^{\nu}}$ corresponding to the box $\Lambda(\mathbf{n})$. For each $\mathbf{n}$ and $\mathbf{x} \in A_{l, \eta}^{c}$ let

$$
\begin{aligned}
C_{\mathbf{x}}^{(\mathbf{n})} & :=\left\{\omega_{\mathbf{n}} \in B_{l}^{(\mathbf{n})}:\left|\frac{1}{\# \Lambda(\mathbf{n})} \log \frac{m_{\mathbf{x}}^{(\mathbf{n})}\left(\omega_{\mathbf{n}}\right)}{p^{(\mathbf{n})}\left(\omega_{\mathbf{n}}\right)}-D_{M, \mathbf{x}}\right|<\delta\right\} \\
& =\left\{\omega_{\mathbf{n}} \in B_{l}^{(\mathbf{n})} \mid e^{\# \Lambda(\mathbf{n}) \cdot\left(D_{M, \mathbf{x}}-\delta\right)}<\frac{m_{\mathbf{x}}^{(\mathbf{n})}\left(\omega_{\mathbf{n}}\right)}{p^{(\mathbf{n})}\left(\omega_{\mathbf{n}}\right)}<e^{\# \Lambda(\mathbf{n}) \cdot\left(D_{M, \mathbf{x}}+\delta\right)}\right\} .
\end{aligned}
$$

The indicator function of $C_{\mathbf{x}}^{(\mathbf{n})}$ corresponds to the projector $q_{\mathbf{x}}^{(\mathbf{n})} \in \mathcal{B}_{l}^{(\mathbf{n})}$. The definition of the set $C_{\mathbf{x}}^{(\mathbf{n})}$ implies a bound on the probability of this set with respect to the probability measure $p$ :

$$
\begin{aligned}
p^{(\mathbf{n})}\left(C_{\mathbf{x}}^{(\mathbf{n})}\right)=\varphi\left(q_{\mathbf{x}}^{(\mathbf{n})}\right) & \leq m_{\mathbf{x}}^{(\mathbf{n})}\left(C_{\mathbf{x}}^{(\mathbf{n})}\right) e^{-\# \Lambda(\mathbf{n}) \cdot\left(D_{M, \mathbf{x}}-\delta\right)} \\
& \leq e^{-\# \Lambda(\mathbf{n}) \cdot\left(D_{M, \mathbf{x}}-\delta\right)} \\
& \leq e^{-\# \Lambda(\mathbf{n}) \cdot(\# \Lambda(l)(s(\psi, \varphi)-\eta)-\delta)}
\end{aligned}
$$

because for all $\mathbf{x} \in A_{l, \eta}^{c}$ we have

$$
\begin{aligned}
D_{M, \mathbf{x}} & \stackrel{\text { by }}{\geq} D\left(m_{\mathbf{x}}^{(1)}, p^{(1)}\right)=S\left(\psi_{\mathbf{x}}^{(\Lambda(l))} \uparrow \mathcal{B}_{l}, \varphi^{(\Lambda(l))} \uparrow \mathcal{B}_{l}\right) \\
& \geq \# \Lambda(l)(s(\psi, \varphi)-\eta),
\end{aligned}
$$

by (201). The limit assertion (22) implies the existence of an $N \in \mathbb{N}$ such that for any $\mathbf{n} \in \mathbb{N}^{\nu}$ with $\Lambda(\mathbf{n}) \supset \Lambda(N)$

$$
m_{\mathbf{x}}^{(\mathbf{n})}\left(C_{\mathbf{x}}^{(\mathbf{n})}\right) \geq 1-\frac{\varepsilon}{2}, \quad \forall \mathbf{x} \in A_{l, \eta}^{c} .
$$

For each $\mathbf{y} \in \mathbb{N}^{\nu}$ with $y_{i} \geq N l$ let $y_{i}=n_{i} l+j_{i}$, where $n_{i} \geq N$ and $0 \leq j_{i}<l$. We set

$$
q_{l \mathbf{n}}:=\bigvee_{\mathbf{x} \in A_{l, \eta}^{c}} q_{\mathbf{x}}^{(\mathbf{n})}
$$

and denote by $q_{\mathbf{y}}$ the embedding of $q_{l \mathbf{n}}$ in $\mathcal{A}^{(\mathbf{y})}$. By (25) and (19) we obtain

$$
\begin{aligned}
\Psi^{(\mathbf{y})}\left(q_{\mathbf{y}}\right) & =\frac{1}{\# \Lambda(k(l))} \sum_{\mathbf{x} \in \Lambda(k(l))} \psi_{\mathbf{x}}^{(\mathbf{y})}\left(q_{\mathbf{y}}\right) \\
& \geq \frac{1}{\# \Lambda(k(l))} \sum_{\mathbf{x} \in \Lambda(k(l))} \psi_{\mathbf{x}}^{(\mathbf{y})}\left(q_{\mathbf{x}}^{(\mathbf{n})}\right) \\
& \geq \frac{1}{\# \Lambda(k(l))} \# A_{l, \eta}^{c} \cdot\left(1-\frac{\varepsilon}{2}\right) \geq 1-\varepsilon .
\end{aligned}
$$


Thus the condition in the definition of $\beta_{\varepsilon, \mathbf{y}}(\psi, \varphi)$ is satisfied. We are now able to estimate $\beta_{\varepsilon, \mathbf{y}}(\psi, \varphi)$ using (23):

$$
\begin{aligned}
\beta_{\varepsilon, \mathbf{y}}(\psi, \varphi) & \leq \log \varphi^{(\mathbf{y})}\left(q_{\mathbf{y}}\right) \\
& \leq \log \sum_{\mathbf{x} \in A_{l, \eta}^{c}} e^{-\# \Lambda(\mathbf{n}) \cdot\left(D_{M, \mathbf{x}}-\delta\right)} \\
& \leq \log \left(\# A_{l, \eta}^{c} \cdot e^{-\# \Lambda(\mathbf{n}) \cdot(\# \Lambda(l)(s(\psi, \varphi)-\eta)-\delta)}\right) \\
& =\log \left(\# A_{l, \eta}^{c}\right)-\# \Lambda(\ln )\left(s(\psi, \varphi)-\eta-\frac{\delta}{\# \Lambda(l)}\right) .
\end{aligned}
$$

This leads to

$$
\limsup _{\Lambda(\mathbf{y}) \nearrow \mathbb{N}^{\nu}} \frac{1}{\# \Lambda(\mathbf{y})} \beta_{\varepsilon, \mathbf{y}}(\psi, \varphi) \leq-s(\psi, \varphi)+\eta+\delta,
$$

since $\# A_{l, \eta}^{c}$ does not depend on $\mathbf{n}$ and $\Lambda(\mathbf{y}) \nearrow \mathbb{N}^{\nu}$ if and only if $\Lambda(\mathbf{n}) \nearrow \mathbb{N}^{\nu}$. Since $\eta, \delta>0$ were chosen arbitrarily we have

$$
\limsup _{\Lambda(\mathbf{n}) \nearrow \mathbb{N}^{\nu}} \frac{1}{\# \Lambda(\mathbf{n})} \beta_{\varepsilon, \mathbf{n}}(\psi, \varphi) \leq-s(\psi, \varphi) .
$$

Suppose we are given a sequence $\left(p_{\mathbf{n}}\right)$ of projectors in $\mathcal{A}^{(\mathbf{n})}$ and a stationary state $\psi$ on $\mathcal{A}^{\infty}$ with mean entropy $s(\psi)$. We consider the positive operators

$$
p_{\mathbf{n}} D_{\psi(\mathbf{n})} p_{\mathbf{n}}=\sum_{i=1}^{d(\mathbf{n})} \lambda_{\mathbf{n}, i} r_{\mathbf{n}, i} \quad d(\mathbf{n}):=\operatorname{tr}\left(p_{\mathbf{n}}\right),
$$

where the numbers $\lambda_{\mathbf{n}, i}$ are the eigenvalues and the $r_{\mathbf{n}, i}$ form a complete set of eigen-projectors of $p_{\mathbf{n}} D_{\psi(\mathbf{n})} p_{\mathbf{n}}$. We set

$$
T_{\mathbf{n}, \delta}:=\left\{i \in\{1, \ldots, d(\mathbf{n})\}: \lambda_{\mathbf{n}, i} \leq e^{-\# \Lambda(\mathbf{n})(s(\psi)-\delta)}\right\} \quad \text { for } \delta>0,
$$

and denote by $T_{\mathbf{n}, \delta}^{c}$ the complement of this set.

Lemma 3.5 Let $\psi$ be an ergodic state on $\mathcal{A}^{\infty}$ with mean entropy $s(\psi)$ and let $\left(p_{\mathbf{n}}\right)$ be a sequence of projectors in $\mathcal{A}^{(\mathbf{n})}$, respectively. If $p_{T_{\mathbf{n}, \delta}^{c}}$ is the projector corresponding to the set $T_{\mathbf{n}, \delta}^{c}$ then

$$
\lim _{\Lambda(\mathbf{n}) \nearrow \mathbb{N}^{\nu}} \psi^{(\mathbf{n})}\left(p_{T_{\mathbf{n}, \delta}^{c}}\right)=0
$$

for all $\delta>0$. 
Proof of Lemma 3.5; We have

$$
1 \geq \sum_{i \in T_{\mathbf{n}, \delta}^{c}} \lambda_{\mathbf{n}, i}>e^{-\# \Lambda(\mathbf{n})(s(\psi)-\delta)} \# T_{\mathbf{n}, \delta}^{c}=e^{-\# \Lambda(\mathbf{n})(s(\psi)-\delta)} \operatorname{tr}\left(p_{T_{\mathbf{n}, \delta}^{c}}\right),
$$

and, consequently

$$
\frac{1}{\# \Lambda(\mathbf{n})} \log \left(\operatorname{tr}\left(p_{T_{\mathbf{n}, \delta}^{c}}\right)\right)<s(\psi)-\delta
$$

If we would have $\psi^{(\Lambda(\mathbf{n}))}\left(p_{T_{\mathbf{n}, \delta_{0}}^{c}}\right) \geq a>0$ for infinitely many $\mathbf{n}$ and some $a>0$ there would be a contradiction to Proposition 2.1 in [1, which implies that there is no sequence $\left(q_{\mathbf{n}}\right)$ of projectors in $\mathcal{A}^{(\mathbf{n})}$ with $\psi^{(\mathbf{n})}\left(q_{\mathbf{n}}\right) \geq a>0$ and $\lim \sup _{\Lambda(\mathbf{n}) \nearrow \mathbb{N}^{\nu}} \frac{1}{\# \Lambda(\mathbf{n})} \log \left(\operatorname{tr}\left(q_{\mathbf{n}}\right)\right)<s(\psi)$.

Lemma 3.6 Let $\psi$ be an ergodic state on $\mathcal{A}^{\infty}$ and let $\varphi$ be a stationary product state on $\mathcal{A}^{\infty}$. Suppose that $s(\psi, \varphi)<\infty$ holds, then for every $\varepsilon \in$ $(0,1)$

$$
\liminf _{\Lambda(\mathbf{n}) \nearrow \mathbb{N}^{\nu}} \frac{1}{\# \Lambda(\mathbf{n})} \beta_{\varepsilon, \mathbf{n}}(\psi, \varphi) \geq-s(\psi, \varphi)
$$

Proof of Lemma 3.6; Let $\left(t_{\mathbf{y}}\right)$ be a sequence of projectors, $t_{\mathbf{y}} \in \mathcal{A}^{(\mathbf{y})}$, with $\Lambda(\mathbf{y}) \nearrow \mathbb{N}^{\nu}$ and

$$
\liminf _{\Lambda(\mathbf{y}) \nearrow \mathbb{N}^{\nu}} \frac{1}{\# \Lambda(\mathbf{y})} \log \varphi^{(\mathbf{y})}\left(t_{\mathbf{y}}\right)<-s(\psi, \varphi)
$$

Then there exists an $a>0$ and a subsequence, which we denote by $\left(t_{\mathbf{y}}\right)$ for notational simplicity, fulfilling

$$
\varphi^{(\mathbf{y})}\left(t_{\mathbf{y}}\right)<e^{-\# \Lambda(\mathbf{y})(s(\psi, \varphi)+a)} .
$$

We consider an integer $l \geq 1$ and the $G_{l}$-ergodic decomposition of $\psi$ :

$$
\psi=\frac{1}{\# \Lambda(\mathbf{k}(l))} \sum_{\mathbf{x} \in \Lambda(\mathbf{k}(l))} \psi_{\mathbf{x}} .
$$

As in the proof of Lemma 3.4 for each $\varepsilon, \eta>0$ we can choose $l$ in such a way that we have

$$
\frac{\# A_{l, \eta}^{c}}{\# \Lambda(\mathbf{k}(l))}\left(1-\frac{\varepsilon}{2}\right) \geq 1-\varepsilon,
$$

where the set $A_{l, \eta}$ was defined by (17). Recall that we have by definition,

$$
\frac{1}{\# \Lambda(l)} S\left(\psi_{\mathbf{x}}^{(l)} \uparrow \mathcal{B}_{l}, \varphi^{(l)} \uparrow \mathcal{B}_{l}\right) \geq s(\psi, \varphi)-\eta \quad \text { for all } \mathbf{x} \in A_{l, \eta}^{c}
$$


We consider again the abelian quasi-local algebra $\mathcal{B}_{l}^{\infty}$, which will be identified with the algebra of continuous functions on the maximal ideal space $B_{l}^{\infty}:=B_{l}^{\mathbb{Z}^{\nu}}$, bearing in mind that the restrictions of the $G_{l}$-ergodic components of $\psi$ and $\varphi$ to this algebra are $G_{l}$-ergodic. We denote those restrictions by $m_{\mathbf{x}}, \mathbf{x} \in \Lambda(\mathbf{k}(l))$, and $p$. As in the proof of Lemma 3.4 we can show that for $\delta>0$ the sets

$$
C_{\mathbf{x}, \delta}^{(\mathbf{n})}:=\left\{\omega_{\mathbf{n}} \in B_{l}^{(\mathbf{n})}: e^{\# \Lambda(\mathbf{n})\left(D_{M, \mathbf{x}}-\frac{\delta}{2}\right)}<\frac{m_{\mathbf{x}}^{(\mathbf{n})}\left(\omega_{\mathbf{n}}\right)}{p^{(\mathbf{n})}\left(\omega_{\mathbf{n}}\right)}<e^{\# \Lambda(\mathbf{n})\left(D_{M, \mathbf{x}}+\frac{\delta}{2}\right)}\right\}
$$

fulfil

$$
\lim _{\Lambda(\mathbf{n}) \nearrow \mathbb{N}^{\nu}} m_{\mathbf{x}}^{(\mathbf{n})}\left(C_{\mathbf{x}, \delta}^{(\mathbf{n})}\right)=1 \quad \text { for all } \mathbf{x} \in \Lambda(\mathbf{k}(l)) .
$$

In a similar way, by employing the classical Shannon-McMillan theorem, we can see that for

$$
F_{\mathbf{x}, \delta}^{(\mathbf{n})}:=\left\{\omega_{\mathbf{n}} \in B_{l}^{(\mathbf{n})}: e^{-\# \Lambda(\mathbf{n})\left(h_{\mathbf{x}}+\frac{\delta}{2}\right)}<m_{\mathbf{x}}^{(\mathbf{n})}\left(\omega_{\mathbf{n}}\right)<e^{-\# \Lambda(\mathbf{n})\left(h_{\mathbf{x}}-\frac{\delta}{2}\right)}\right\},
$$

we have

$$
\lim _{\Lambda(\mathbf{n}) \nearrow \mathbb{N}^{\nu}} m_{\mathbf{x}}^{(\mathbf{n})}\left(F_{\mathbf{x}, \delta}^{(\mathbf{n})}\right)=1 \quad \text { for all } \mathbf{x} \in \Lambda(\mathbf{k}(l)),
$$

where $h_{\mathbf{x}}$ denotes the Shannon entropy rate of $m_{\mathbf{x}}$. Each $\omega_{\mathbf{n}} \in B_{l}^{(\mathbf{n})}$ corresponds to a minimal projector $q_{\mathbf{n}} \in \mathcal{B}_{l}^{(\mathbf{n})} \subset \mathcal{A}^{(l \mathbf{n})}$. So, for all $\mathbf{x} \in A_{l, \eta}^{c}$ and for any $\omega_{\mathbf{n}} \in C_{\mathbf{x}, \delta}^{(\mathbf{n})} \cap F_{\mathbf{x}, \delta}^{(\mathbf{n})}$ and corresponding $q_{\mathbf{n}}$ we have

$$
\begin{aligned}
\varphi^{(l \mathbf{n})}\left(q_{\mathbf{n}}\right) & =p^{(\mathbf{n})}\left(\omega_{\mathbf{n}}\right) \\
& >e^{-\# \Lambda(\mathbf{n})\left(D_{M, \mathbf{x}}+h_{\mathbf{x}}+\delta\right)} \\
& \geq e^{-\# \Lambda(\mathbf{n})\left(s(\psi, \varphi) l^{\nu}+h_{\mathbf{x}}+\delta\right)} .
\end{aligned}
$$

In fact, the first inequality follows from the definitions of the sets $C_{\mathbf{x}, \delta}^{(\mathbf{n})}$ and $F_{\mathbf{x}, \delta}^{(\mathbf{n})}$ and the assumption that $\omega_{\mathbf{n}} \in C_{\mathbf{x}, \delta}^{(\mathbf{n})} \cap F_{\mathbf{x}, \delta}^{(\mathbf{n})}$. The second inequality is a consequence of (21). Moreover, by (24) we get the upper estimate

$$
\begin{aligned}
\varphi^{(l \mathbf{n})}\left(q_{\mathbf{n}}\right) & =p^{(\mathbf{n})}\left(\omega_{\mathbf{n}}\right) \\
& <e^{-\# \Lambda(\mathbf{n})\left(D_{M, \mathbf{x}}+h_{\mathbf{x}}-\delta\right)} \\
& \leq e^{-\# \Lambda(\mathbf{n})\left((s(\psi, \varphi)-\eta) l^{\nu}+h_{\mathbf{x}}-\delta\right)} .
\end{aligned}
$$

Next, observe that the representation (13) of $\mathcal{B}_{l}$ implies that

$$
D_{\psi_{\mathbf{x}}^{(l)} \mathcal{B}_{l}}=\sum q_{l, i} D_{\psi_{\mathbf{x}}^{(l)}} q_{l, i},
$$


where $\left(q_{l, i}\right)$ is a complete set of minimal eigen-projectors of $D_{\varphi^{(l)}}$. We get

$$
\begin{aligned}
\operatorname{tr}\left(D_{\psi_{\mathbf{x}}^{(l)} \mathcal{B}_{l}} \log D_{\varphi^{(l)}}\right) & =\operatorname{tr}\left(\sum q_{l, i} D_{\psi_{\mathbf{x}}^{(l)}} q_{l, i} \log D_{\varphi^{(l)}}\right) \\
& =\operatorname{tr}\left(D_{\psi_{\mathbf{x}}^{(l)}} \sum q_{l, i}\left(\log D_{\varphi^{(l)}}\right) q_{l, i}\right) \\
& =\operatorname{tr}\left(D_{\psi_{\mathbf{x}}^{(l)}} \log D_{\varphi^{(l)}}\right) .
\end{aligned}
$$

Finally, by our assumption that $s(\psi, \varphi)<\infty$ holds we know that $s(\psi, \varphi)=$ $-s(\psi)-\operatorname{tr}\left(D_{\psi^{(1)}} \log D_{\varphi^{(1)}}\right)$. Using the product structure of the state $\varphi$ and the fact just derived we obtain

$$
\begin{array}{rll}
S\left(\psi_{\mathbf{x}}^{(l \mathbf{n})} \uparrow \mathcal{B}_{l}^{(\mathbf{n})}, \varphi^{(l \mathbf{n})} \uparrow \mathcal{B}_{l}^{(\mathbf{n})}\right) & = & -H\left(m_{\mathbf{x}}^{(\mathbf{n})}\right)-\operatorname{tr}\left(D_{\psi_{\mathbf{x}}^{(\mathbf{n})} \uparrow \mathcal{B}_{l}^{\mathbf{n}}} \log D_{\varphi^{(l \mathbf{n})}}\right) \\
& = & -H\left(m_{\mathbf{x}}^{(\mathbf{n})}\right)-\# \Lambda(\mathbf{n}) \operatorname{tr}\left(D_{\psi_{\mathbf{x}}^{(l)} \uparrow \mathcal{B}_{l}} \log D_{\varphi^{(l)}}\right) \\
& (\text { by } \stackrel{(34)}{=}) & -H\left(m_{\mathbf{x}}^{(\mathbf{n})}\right)-\# \Lambda(\mathbf{n})\left(\operatorname{tr}\left(D_{\psi_{\mathbf{x}}^{(l)}} \log D_{\varphi^{(l)}}\right) .\right.
\end{array}
$$

On the other hand we have for all $\mathbf{x} \in A_{l, \eta}^{c}$

$$
\begin{aligned}
S\left(\psi_{\mathbf{x}}^{(l \mathbf{n})} \uparrow \mathcal{B}_{l}^{(\mathbf{n})}, \varphi^{(l \mathbf{n})} \uparrow \mathcal{B}_{l}^{(\mathbf{n})}\right) \geq & \# \Lambda(\mathbf{n})\left(-H\left(m_{\mathbf{x}}^{(1)}\right)-\operatorname{tr}\left(D_{\psi_{\mathbf{x}}^{(l)} \uparrow \mathcal{B}_{l}} \log D_{\varphi^{(l)}}\right)\right) \\
& (\text { by the subadditivity of the entropy) } \\
= & \# \Lambda(\mathbf{n}) S\left(\psi_{\mathbf{x}}^{(l)} \uparrow \mathcal{B}_{l}, \varphi^{(l)} \uparrow \mathcal{B}_{l}\right) \\
\geq & \# \Lambda(\mathbf{n}) l^{\nu}(s(\psi, \varphi)-\eta) \\
& \left(\text { by the choice of algebra } \mathcal{B}_{l}\right) \\
= & \# \Lambda(\mathbf{n}) l^{\nu}\left(-s(\psi)-\operatorname{tr}\left(D_{\psi^{(1)}} \log D_{\varphi^{(1)}}\right)-\eta\right) .
\end{aligned}
$$

The equation chain (35) and the inequality chain (36) imply that

$$
\begin{aligned}
-H\left(m_{\mathbf{x}}^{(\mathbf{n})}\right) & -\# \Lambda(\mathbf{n})\left(\operatorname{tr}\left(D_{\psi_{\mathbf{x}}^{(l)}} \log D_{\varphi^{(l)}}\right)\right) \\
& \geq \# \Lambda(\mathbf{n}) l^{\nu}\left(-s(\psi)-\operatorname{tr}\left(D_{\psi^{(1)}} \log D_{\varphi^{(1)}}\right)-\eta\right) .
\end{aligned}
$$

Note that the third and the fourth item of Theorem 3.1] show that

$$
\operatorname{tr}\left(D_{\psi_{\mathbf{x}}^{(l)}} \log D_{\varphi^{(l)}}\right)=l^{\nu} \operatorname{tr}\left(D_{\psi^{(1)}} \log D_{\varphi^{(1)}}\right),
$$

in the case where $s(\psi, \varphi)<\infty$. Dividing (37) by $-\# \Lambda(\mathbf{n})$ and taking the limit $\Lambda(\mathbf{n}) \nearrow \mathbb{N}^{\nu}$ leads to

$$
l^{\nu} s(\psi) \leq h_{\mathbf{x}} \leq l^{\nu}(s(\psi)+\eta)
$$

where the lower bound simply follows from the fact that the entropy on a maximally abelian subalgebra is not less than the entropy on the algebra. This inequality, (32) and (33) imply:

$$
e^{-\# \Lambda(l \mathbf{n})\left(s(\psi, \varphi)+s(\psi)+\eta+\delta / l^{\nu}\right)} \leq \varphi^{(l \mathbf{n})}\left(q_{\mathbf{n}}\right) \leq e^{-\# \Lambda(l \mathbf{n})\left(s(\psi, \varphi)+s(\psi)-\eta-\delta / l^{\nu}\right)} .
$$


Let $p_{l \mathbf{n}}$ denote the projector corresponding to the set $\bigcup_{\mathbf{x} \in A_{l, \eta}^{c}} C_{\mathbf{x}, \delta}^{(\mathbf{n})} \cap F_{\mathbf{x}, \delta}^{(\mathbf{n})}$ and $p_{\mathbf{n}, \mathbf{x}}$ be the projector which corresponds to $C_{\mathbf{x}, \delta}^{(\mathbf{n})} \cap F_{\mathbf{x}, \delta}^{(\mathbf{n})}$. For sufficiently large $\mathbf{n} \in \mathbb{N}^{\nu}$ we have

$$
\psi_{\mathbf{x}}\left(p_{\mathbf{n}, \mathbf{x}}\right) \geq 1-\frac{\varepsilon}{2} \quad \text { by (30) and (31) }
$$

and hence by (29)

$$
\begin{aligned}
\psi\left(p_{l \mathbf{n}}\right) & \geq \frac{1}{\# \Lambda(\mathbf{k}(l))} \sum_{\mathbf{x} \in A_{l, \eta}^{c}} \psi_{\mathbf{x}}\left(p_{\mathbf{n}, \mathbf{x}}\right) \\
& \geq \frac{1}{\# \Lambda(\mathbf{k}(l))} \# A_{l, \eta}^{c}\left(1-\frac{\varepsilon}{2}\right) \geq 1-\varepsilon .
\end{aligned}
$$

Any $t_{\mathbf{y}}$ fulfilling (28) can be embedded in an appropriately chosen $\mathcal{A}^{(l \mathbf{n})}$. Indeed, choose the unique $\mathbf{n} \in \mathbb{N}^{\nu}$ such that $\left(n_{i}-1\right) l \leq y_{i}<n_{i} l$ for all $i \in\{1, \ldots, \nu\}$ and set

$$
e_{l \mathbf{n}}:=t_{\mathbf{y}} \otimes \mathbf{1}_{\Lambda(l \mathbf{n}) \backslash \Lambda(\mathbf{y})} .
$$

Then we have

$$
\psi^{(l \mathbf{n})}\left(e_{l \mathbf{n}}\right)=\psi^{(\mathbf{y})}\left(t_{\mathbf{y}}\right),
$$

and

$$
\begin{aligned}
\psi^{(l \mathbf{n})}\left(e_{l \mathbf{n}}\right) & =\psi^{(l \mathbf{n})}\left(e_{l \mathbf{n}} p_{l \mathbf{n}}\right)+\psi^{(l \mathbf{n})}\left(e_{l \mathbf{n}}\left(\mathbf{1}-p_{l \mathbf{n}}\right)\right) \\
& \leq \psi^{(\mathbf{l n})}\left(e_{l \mathbf{n}} p_{l \mathbf{n}}\right)+\varepsilon \quad(\text { by (40) }) .
\end{aligned}
$$

Applying this argument once more we obtain

$$
\begin{aligned}
\psi^{(l \mathbf{n})}\left(e_{l \mathbf{n}}\right) & \leq \psi^{(l \mathbf{n})}\left(p_{l \mathbf{n}} e_{l \mathbf{n}} p_{l \mathbf{n}}\right)+2 \varepsilon \\
& =\operatorname{tr}\left(p_{l \mathbf{n}} D_{\psi^{(l \mathbf{n})}} p_{l \mathbf{n}} e_{l \mathbf{n}}\right)+2 \varepsilon .
\end{aligned}
$$

In the final step we will prove that the first term in the last line above can be made arbitrarily small. Using the notation from (26), (27) and applying Lemma 3.5 we know that

$$
\begin{aligned}
\operatorname{tr}\left(p_{l \mathbf{n}} D_{\psi^{(l \mathbf{n})}} p_{l \mathbf{n}} e_{l \mathbf{n}}\right) & \leq e^{-\# \Lambda(l \mathbf{n})(s(\psi)-\delta)} \sum_{i \in T_{l \mathbf{n}, \delta}} \operatorname{tr}\left(r_{l \mathbf{n}, i} e_{l \mathbf{n}}\right)+\varepsilon \\
& \leq e^{-\# \Lambda(l \mathbf{n})(s(\psi)-\delta)} \sum_{i=1}^{\operatorname{tr}\left(p_{l \mathbf{n}}\right)} \operatorname{tr}\left(r_{l \mathbf{n}, i} e_{l \mathbf{n}}\right)+\varepsilon \\
& =e^{-\# \Lambda(l \mathbf{n})(s(\psi)-\delta)} \operatorname{tr}\left(p_{l \mathbf{n}} e_{l \mathbf{n}}\right)+\varepsilon
\end{aligned}
$$

for sufficiently large $\mathbf{n} \in \mathbb{N}^{\nu}$. We represent the projector $p_{l \mathbf{n}}$ as a sum of unique minimal projections in $\mathcal{B}_{l}^{(\mathbf{n})}$ :

$$
p_{l \mathbf{n}}=\sum_{i=1}^{\operatorname{tr}\left(p_{l \mathbf{n}}\right)} q_{\mathbf{n}, i}
$$


Hence by (39) we have

$$
\begin{aligned}
\operatorname{tr}\left(p_{l \mathbf{n}} e_{l \mathbf{n}}\right) & \leq e^{\# \Lambda(l \mathbf{n})\left(s(\psi, \varphi)+s(\psi)+\eta+\delta / l^{\nu}\right)} \sum_{i=1}^{\operatorname{tr}\left(p_{l \mathbf{n}}\right)} \varphi^{(l \mathbf{n})}\left(q_{\mathbf{n}, i}\right) \operatorname{tr}\left(q_{\mathbf{n}, i} e_{l \mathbf{n}}\right) \\
& =e^{\# \Lambda(l \mathbf{n})\left(s(\psi, \varphi)+s(\psi)+\eta+\delta / l^{\nu}\right)} \varphi^{(l \mathbf{n})}\left(p_{l \mathbf{n}} e_{l \mathbf{n}}\right) \\
& \leq e^{\# \Lambda(\mathbf{l n})\left(s(\psi, \varphi)+s(\psi)+\eta+\delta / l^{\nu}\right)} \varphi^{(\mathbf{y})}\left(t_{\mathbf{y}}\right) \\
& \leq e^{\# \Lambda(\mathbf{l n})\left(s(\psi, \varphi)+s(\psi)+\eta+\delta / l^{\nu}\right)} e^{-\# \Lambda(\mathbf{y})(s(\psi, \varphi)+a)}(\text { by (28) }),
\end{aligned}
$$

since the minimal projectors $q_{\mathbf{n}, i}$ correspond to the eigen-vectors of $D_{\varphi^{(l \mathbf{n})}}$. Inserting this into (43) we obtain

$$
\operatorname{tr}\left(p_{l \mathbf{n}} D_{\psi(l \mathbf{n})} p_{l \mathbf{n}} e_{l \mathbf{n}}\right) \leq e^{-\# \Lambda(l \mathbf{n})\left(\left(\frac{\# \Lambda(\mathbf{y})}{\# \Lambda(\mathbf{n})}-1\right) s(\psi, \varphi)+\frac{\# \Lambda(\mathbf{y})}{\# \Lambda(\mathbf{n})} a-\delta-\eta-\frac{\delta}{l^{\nu}}\right)}+\varepsilon .
$$

Note that $\lim _{\Lambda(\mathbf{y}) \nearrow \mathbb{N}^{\nu}} \frac{\# \Lambda(\mathbf{y})}{\# \Lambda(l \mathbf{n})}=1$, and that $a>0$. Hence if we choose $\delta, \eta$ small enough and $\mathbf{n}$ large enough we can achieve that the exponent in the last inequality becomes negative eventually. This inequality toghether with (42) and (41) shows that

$$
\lim _{\Lambda(\mathbf{y}) \nearrow \mathbb{N}^{\nu}} \psi\left(t_{\mathbf{y}}\right)=0
$$

The combination of Lemma 3.4 and Lemma 3.6 yields the assertion of Theorem 2.1. To derive Theorem 2.2. consider the projectors $p_{l \mathbf{n}}$ constructed in the proof of Lemma 3.6. They can be written as the sum of minimal projectors $q_{\mathbf{n}}$ fulfilling (39). From the fact, that these are minimal eigen-projectors for $D_{\varphi(\mathbf{n})}$ we derive that (39) is valid even if we replace $q_{\mathbf{n}}$ by any minimal projector which is dominated by $p_{l \mathbf{n}}$. So we see that, if we would choose the projectors $p_{\mathbf{n}}(\varepsilon)$ to be specified for Theorem 2.2 just as $p_{l \mathbf{n}}$ for boxes with edge lengths being multiples of $l$, item 3 would be satisfied for large $\mathbf{n}$, supposed $l$ is (fixed but) large enough. Item 1 is fulfilled for large $\mathbf{n}$ in view of (40). Observe now that embedding the projectors $p_{l \mathbf{n}}$ into $\mathcal{A}^{(\mathbf{y})}$ for $0 \leq \mathbf{y}-l \mathbf{n}<(l, l, \ldots, l)$ leads us to a family of projectors $\left(p_{\mathbf{n}}\right)$ still fulfilling item 1 . Item 3 is satisfied by this family, too, since $\varphi^{(1)}$ was supposed faithful. In order to ensure that item 2 is fulfilled, the constructed family of projectors $\left(p_{\mathbf{n}}\right)$ has to be modified. Represent $p_{\mathbf{n}}$ as a sum of eigen-projectors of the operator $p_{\mathbf{n}} D_{\psi^{(\mathbf{n})}} p_{\mathbf{n}}$. Now from the definition of the sets $F_{\mathbf{x}, \delta}^{(\mathbf{n})}$ we easily conclude that $\operatorname{tr}\left(p_{\mathbf{n}}\right)<e^{\# \Lambda(\mathbf{n})(s(\psi)+\varepsilon)}$ can be guaranteed for large $\mathbf{n}$. So the asymptotic contribution to $\psi^{(\mathbf{n})}\left(p_{\mathbf{n}}\right)$ of eigen-values of $p_{\mathbf{n}} D_{\psi^{(\mathbf{n})}} p_{\mathbf{n}}$ of magnitude exponentially smaller than $e^{-\# \Lambda(\mathbf{n})(s(\psi)+\varepsilon)}$ can be neglected. The asymptotic contribution to $\psi^{(\mathbf{n})}\left(p_{\mathbf{n}}\right)$ of eigen-values of $p_{\mathbf{n}} D_{\psi(\mathbf{n})} p_{\mathbf{n}}$ of magnitude exponentially larger than $e^{-\# \Lambda(\mathbf{n})(s(\psi)-\varepsilon)}$ can be neglected, too, because of Lemma 3.5. So we may omit the eigen-projectors 
corresponding to either too large or too small eigen-values from the sum, getting a modified family $\left(p_{\mathbf{n}}^{\prime}\right)$, which additionally fulfils item 2 . This proves Theorem 2.2.

Acknowledgements We are very thankful to our dear colleagues Tyll Krüger, Ruedi Seiler and Arleta Szkoła for their constant interest and encouragement during the preparation of this article and for many very helpful comments and improvements.

This work was supported by the DFG via SFB 288 'Differentialgeometrie und Quantenphysik' at TU Berlin.

\section{References}

[1] I. Bjelaković, T. Krüger, Ra. Siegmund-Schultze, A. Szkoła, The Shannon-McMillan Theorem for Ergodic Quantum Lattice Systems, arXiv.org, math.DS/0207121, to appear in Inventiones Mathematicae (2003)

[2] O.Bratteli, D.W. Robinson, Operator Algebras and Quantum Statistical Mechanics I, Springer, New York 1979

[3] F. Hiai, D. Petz, The Proper Formula for Relative Entropy and its Asymptotics in Quantum Probability, Commun. Math. Phys. 143, 99$114(1991)$

[4] T. Ogawa, H. Nagaoka, Strong Converse and Stein's Lemma in Quantum Hypothesis Testing, IEEE Trans. Inform. Theo., vol. 46, No. 7, 2428-2433 (2000)

[5] M. Ohya, D. Petz, Quantum Entropy and its Use, Springer, Berlin 1993

[6] D. Ornstein, B. Weiss, The Shannon-McMillan-Breiman Theorem for a Class of Amenable Groups, Israel J. Math. 44 (1), 53-60 (1983)

[7] D. Ruelle, Statistical Mechanics, W.A. Benjamin, New York 1969

[8] P. C. Shields, Two divergence-rate counterexamples, J. Theor. Prob. vol. 6 521-545 (1993) 